\title{
The Bazar Ophiolite of NW Iberia: a relic of the lapetus-Tornquist Ocean in the Variscan suture
}

\author{
Sonia S. Martínez, ${ }^{1}$ Axel Gerdes, ${ }^{2}$ Ricardo Arenas ${ }^{1}$ and Jacobo Abati ${ }^{1}$ \\ ${ }^{1}$ Departamento de Petrología y Geoquimica e Instituto de Geociencias (CSIC), Universidad Complutense, 28040 Madrid, Spain; ${ }^{2}$ Institut für \\ Geowissenschaften, Goethe Universität, D-6438 Frankfurt am Main, Germany
}

\begin{abstract}
The Bazar Ophiolite, one of the ophiolitic units involved in the Variscan suture of NW Iberia, is mainly formed by metagabbroic high $\mathrm{T}$ amphibolites with N-MORB affinity. The ophiolite appears accreted under an arc-derived upper terrane affected by intermediate-P granulite facies metamorphism dated at $496-$ $484 \mathrm{Ma}$. U-Th- $\mathrm{Pb}$ geochronology and Lu-Yb-Hf isotope geochemistry of zircons allow recognizing two growth stages. The first occurred during crystallization of the gabbroic protolith and has been dated at $495 \pm 2 \mathrm{Ma}$, whereas the second one,
\end{abstract}

interpreted as dating the high $\mathrm{T}$ metamorphism, yielded an age of $475 \pm 2 \mathrm{Ma}$. The chronology of the Bazar Ophiolite and its accretionary history suggest that this unit is a relic of the Cambrian ocean located to the North of Gondwana, the lapetus-Tornquist Ocean, accreted to a dissected arc during or before the early stages in the opening of the Rheic Ocean.

\section{Introduction}

The Variscan Belt of NW Iberia shows an impressive section of the suture zone of this orogen, developed in Carboniferous times by the collision of Gondwana and Laurussia during the final event of the Pangea assembly (Matte, 2001; Martínez Catalán et al., 2009). The suture zone appears transported in several allochthonous complexes of which the Órdenes Complex has the largest size and includes the most varied group of exotic terranes (Fig. 1a). An arcderived terrane generated in Cambrian times in the periphery of Gondwana appears in the uppermost position of the allochthonous pile (upper units of NW Iberia). During a subsequent event, this arc rifted from the main continent, drifted to the North and was finally accreted to the southern margin of Laurussia (Gómez Barreiro et al., 2007; Díaz García et al., 2010; Fuenlabrada et al., 2010). A first metamorphic event affecting the arc terrane, with intermediate-P features and dated in the range $496-482 \mathrm{Ma}$, is considered related to the internal dynamics of the peri-Gondwanan arc

Correspondence: Sonia S. Martínez, Dpto. Petrología y Geoquímica, Universidad Complutense de Madrid. Jose Antonio Nováis 2, 28040, Madrid, Spain. Tel.: +34 618544373; e-mail: s.sanchez@geo. ucm.es
(Abati et al., 1999, 2007), whereas a second high-P and high-T event dated at c. $400-390 \mathrm{Ma}$ developed during the collision with Laurussia (Ordoñez Casado et al., 2001; Fernández-Suárez et al., 2007). The arc-derived terrane is thrust over a group of different ophiolitic units (Fig. 1a). The ophiolites in turn thrust a lower terrane with high$\mathrm{P}$ and intermediate- $\mathrm{T}$ metamorphism dated at c. $370 \mathrm{Ma}$ (basal units of NW Iberia; Fig. 1a), interpreted as the edge of the Gondwanan margin subducted under Laurussia during the first stages of the collision, uplifted and incorporated to the allochthonous pile later on (Arenas et al., 1995; Martínez Catalán et al., 1996; Abati et al., 2010).

The ophiolites outlining the Variscan suture were in general formed inside the Rheic Ocean domain, but they have typical compositions of island-arc tholeiites (Sánchez Martínez, 2009). Some of them, as the Vila de Cruces Ophiolite (Fig. 1a; Arenas et al., 2007), were generated in the first stages of the opening of this ocean during Middle Cambrian times, due to the spreading of a back-arc basin. However, the most common ophiolites along the European Variscan Belt have shown ages at around c. $395 \mathrm{Ma}$, and their generation seems to be connected to the activity of an intra-Rheic subduction zone responsible for the main contractional event in this ocean. The Careón Ophiolite is the most representative supra-subduction zone ophiolite in NW Iberia (Fig. 1a; Díaz García et al., 1999; Sánchez Martínez et al., 2007). Nevertheless, there is an ophiolitic unit in the NW of the Iberian Massif, the Bazar Ophiolite (Fig. 1a), which shows lithological constitution and tectonothermal evolution distinctive and different from those shown by the other ophiolitic units. This article presents new U-Pb$\mathrm{Hf}$ and Lu-Yb-Hf zircon data from the metagabbroic amphibolites of the Bazar Ophiolite. These new data, together with the chemical composition of the metagabbroic rocks and their metamorphic evolution, are not compatible with the generation of this ophiolite within the context of the Rheic Ocean, but they better suggest an origin related to a different older oceanic realm.

\section{The Bazar Ophiolite}

The Bazar Ophiolite is located in the westernmost part of the Ordenes Complex (Fig. 1a). It consists of an imbricate of tectonic slices, mainly constituted by metagabbroic rocks and a minor proportion of ultramafic rocks at the base of the unit (Díaz García, 1990). The main tectonic slice (Carballo - Bazar) is c. $4000 \mathrm{~m}$ thick and is composed of amphibolites and foliated metagabbros with high $\mathrm{T}$ foliation, which evolved from an initial granulite-facies tectonothermal event. 


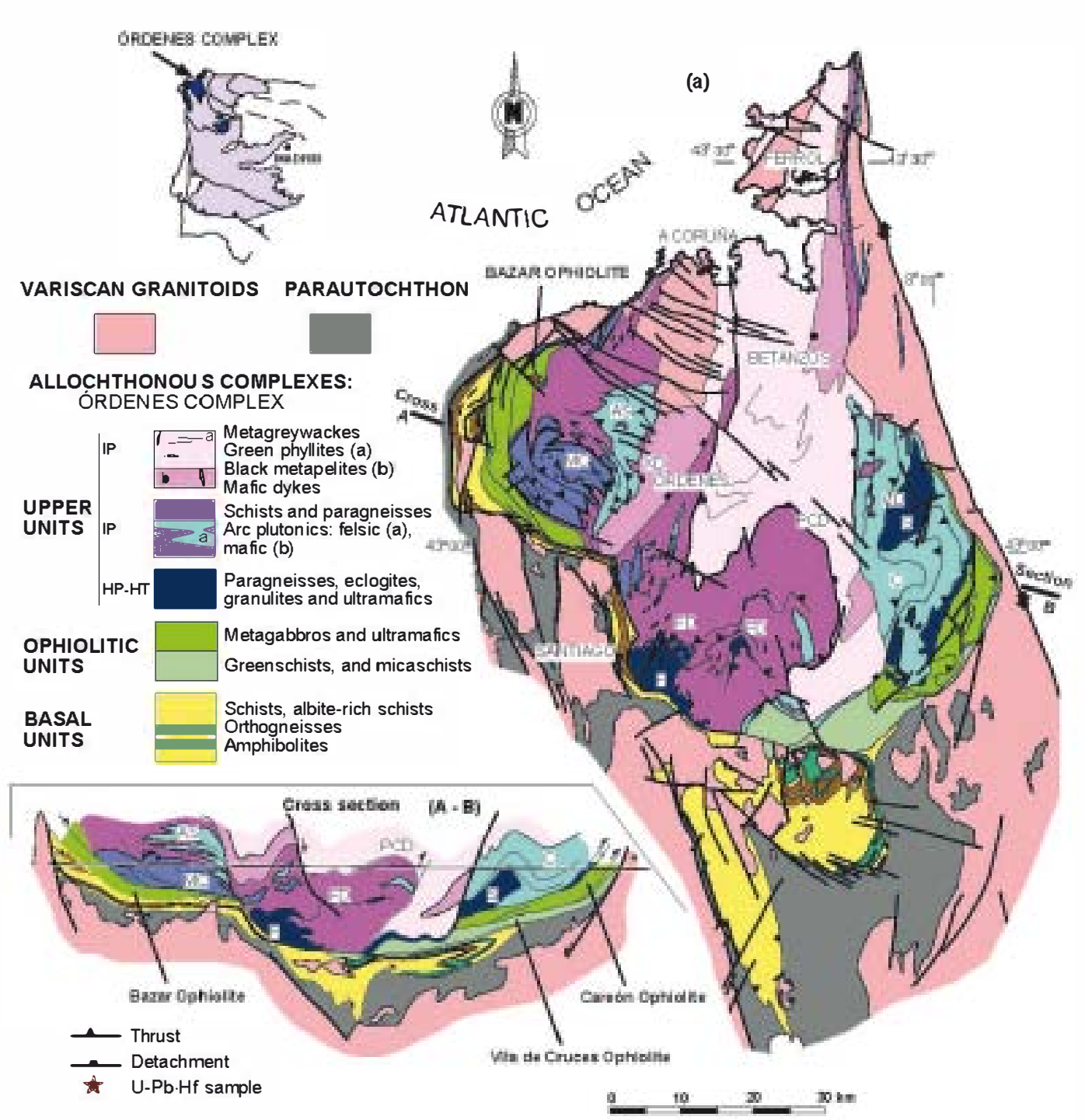

(b)

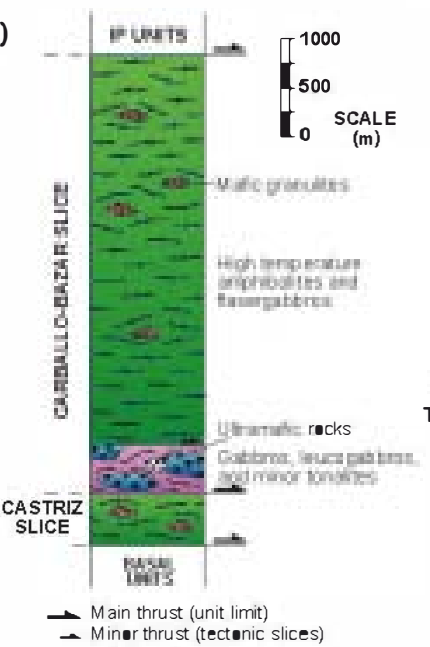

(c)
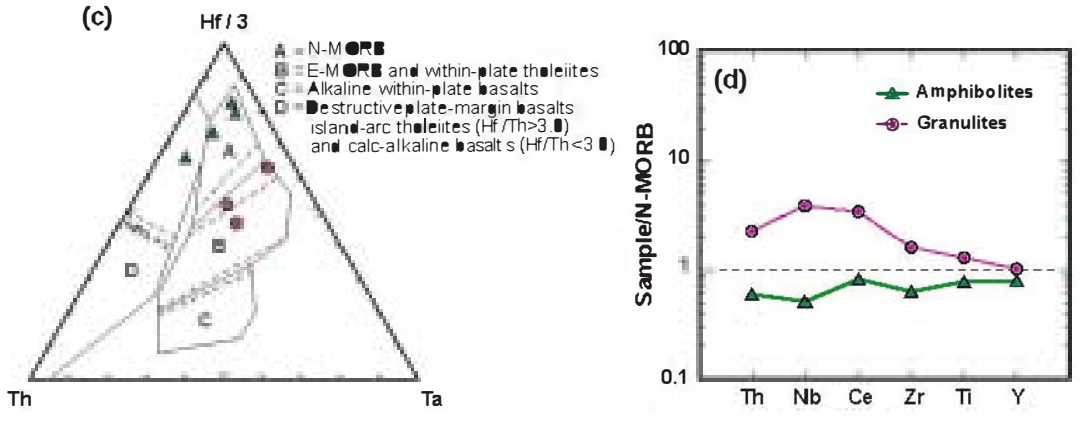

Fig. 1 (a) Geological map and cross-section of the Órdenes Complex with location of the Bazar Ophiolite and other ophiolitic units involved in the Variscan suture. (b) Representative section of the Bazar Ophiolite. (c) Th-Hf-Ta diagram (Wood, 1980) with projection of the metagabbroic amphibolites representing the most common lithology in the ophiolite, and the mafic granulites constuting uncommon minor bodies within the unit. (d) Normal-mid-ocean-ridge basalts (N-MORB) normalized race-element patterns (average composition); selected elements and normalized values after Pearce (1996). 
Scarce metric-sized boudins of mafic granoblastic granulites appear preserved within the metagabbros (Fig. 1b). These boudins appear wrapped by the high $\mathrm{T}$ foliation and their mineral associations indicate lowto-intermediate pressure conditions (plagioclase + clinopyroxene + orthopiroxene + hornblende + ilmenite \pm garnet \pm olivine). The lower section of the main slice has a different lithological composition, as mentioned before, which consists of relatively wellpreserved gabbros and ultramafic rocks, with minor leucogabbros and tonalites (Fig. 1b). The granulite facies event is not recorded in this lower slice, which only shows an amphibolite facies metamorphism.

The geochemical features of the most representative lithologies of the Bazar Ophiolite appeared to be quite complex (Sánchez Martínez, 2009). Regarding the common amphibolites, which represent the most abundant lithological type, they show compositions equivalent to N-MORB (normalmid-ocean ridge basalt, Fig. 1c, d) pointing to a tectonic setting possibly related to a divergent plate margin.
There seems to be no evidence indicating some influence of a subduction zone in the generation of these gabbros unlike what has been registered in the other ophiolitic units described in NW Iberia (Sánchez Martínez et al., 2009). However, the mafic granulites are transitional between MOR (mid-ocean ridge) and WP (within-plate) basalts with normalized trace element patterns similar to those of T-MORB generated in plume ridge interactions (Pearce, 1996).

\section{Results of U-Th-Pb and Lu-Yb-Hf isotope analyses}

From the amphibolite sample $\mathrm{GCH}$ 4-3, located at the top of the main tectonic slice (Fig. la), 71 zircon grains were analysed by U-Th- $\mathrm{Pb}$ LA-ICP-MS at the Goethe University Frankfurt (GUF), obtaining a set of 99 data (Fig. 2b; Table 1). Furthermore, 77 Hf isotope analyses were obtained in the same grains and crystal domains where the U-Th-Pb analyses were performed (Fig. 2d-f; Table 2). The analytical techniques applied for U-Th-Pb and Lu-Yb-Hf isotope determination are described in detail by Gerdes and Zeh (2006, 2009). The studied sample is one of the most representative metabasites of nematoblastic type of this mafic unit constituted by hornblende + plagioclase + clinozoisite + ilmenite + titanite + quartz. The zircon grains were subrounded and may reflect resorption of originally irregular and fragmented zircons, which are typical of mafic rocks. (Corfu et al., 2003). These resorption processes frequently occur in zircons of high-grade metamorphic rocks (Hoskin and Black, 2000). Their size ranges between 100 and $300 \mu \mathrm{m}$ and cathodoluminescence (CL) images were taken from all of them prior to their analysis to observe their internal structure. They show a combination of sector and fine oscillatory zoning, features that can be considered of igneous origin, and many of them also display irregular overgrowths or recrystallized domains lacking internal structure, which would be developed during the granulite-facies metamorphic event undergone by the ophiolitic unit (Fig. 2a).
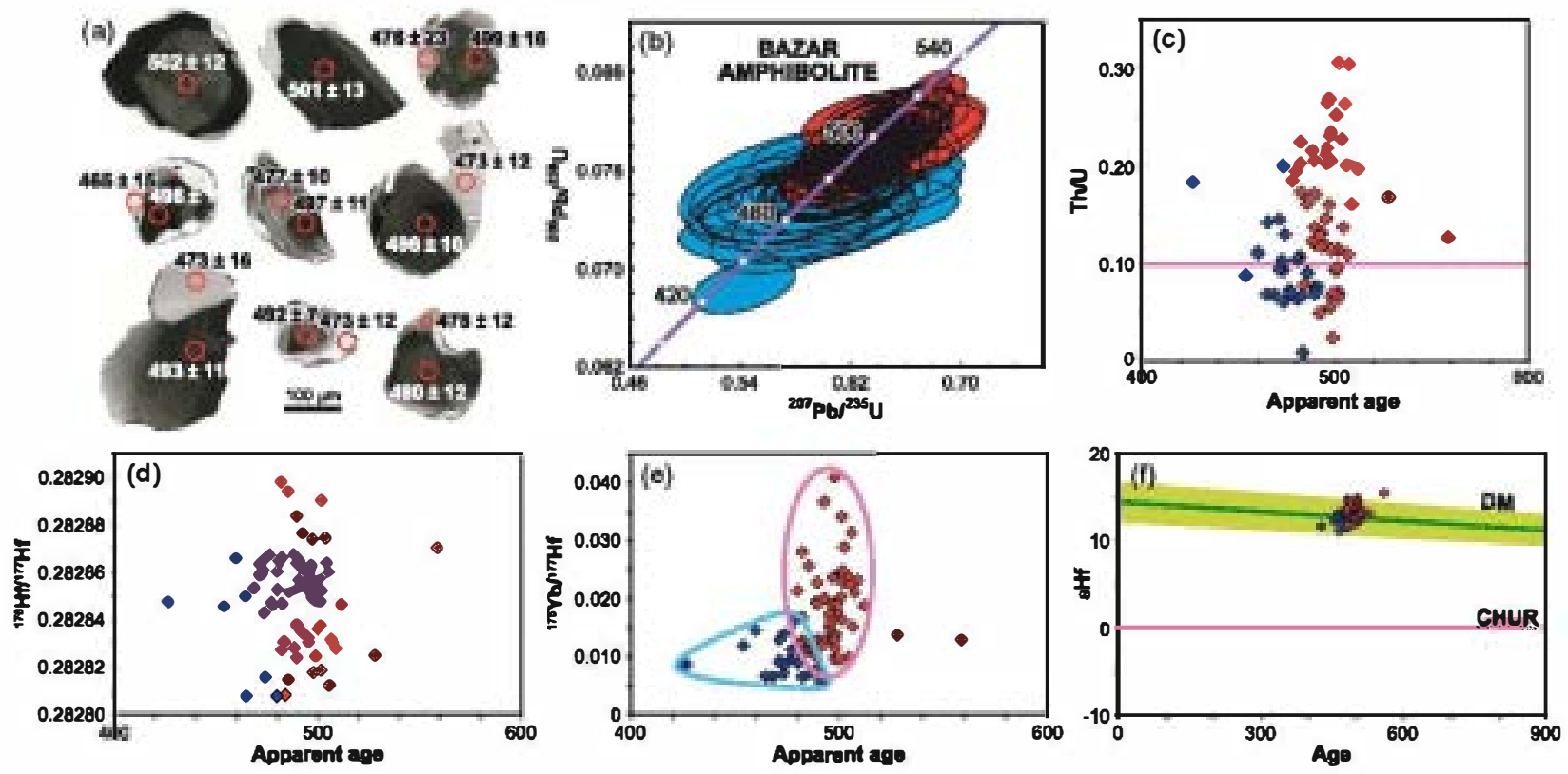

Fig. 2 (a) Cathodo-luminescence images of selected zircons of the Bazar amphibolite GCH-4-3, circles representing the spot size of U-Th-Pb analyses. (b) Concordia diagram showing the results of the U-Pb analyses, with two statistically coherent groups of data. (c) Th/U ratio versus apparent age diagram. (d) Initial ${ }^{176} \mathrm{Hf} /{ }^{177} \mathrm{Hf}$ versus apparent age diagram. (e) ${ }^{176} \mathrm{Yb} /{ }^{177} \mathrm{Hf}$ versus apparent age diagram, the different acends of each group of analyses appearing encircled. (f) $\varepsilon \mathrm{Hf}$ versus apparent age plot; the depleted mantle array (DM) is extrapolated from average modern-day values of mid-ocean ridge basalts (Chauvel and BlichertToft, 2001), assuming a linear behaviour from $\varepsilon \mathrm{Hf}=2$ at $4000 \mathrm{Ma}$ (Vervoort and Blichert-Toft, 1999). In all diagrams, red symbols $=$ ircon cores; blue symbols $=$ ircon overgrowths recrystallized domains. 
Table 1 Results of U-Th-Pb LA-ICP-MS analyses of arcon from the sample of Bazar amphibolite.

\begin{tabular}{|c|c|c|c|c|c|c|c|c|c|c|c|c|c|c|c|c|c|c|c|c|}
\hline \multirow[b]{2}{*}{ Grain } & \multirow[b]{2}{*}{ L-No. } & \multirow[b]{2}{*}{$\begin{array}{l}{ }^{207} \mathrm{~Pb}^{*} \\
\text { (cps) }\end{array}$} & \multirow[b]{2}{*}{$\begin{array}{l}\text { Uf } \\
(\mathrm{ppm})\end{array}$} & \multirow[b]{2}{*}{$\begin{array}{l}\mathrm{Pb} \dagger \\
(\mathrm{ppm})\end{array}$} & \multirow[b]{2}{*}{$\begin{array}{l}\text { Th/ } \dagger \\
u\end{array}$} & \multirow[b]{2}{*}{$\begin{array}{l}{ }^{206} \mathrm{~Pb} / \\
{ }^{204} \mathrm{~Pb}\end{array}$} & \multirow[b]{2}{*}{$\begin{array}{l}{ }^{206} \mathrm{~Pb} / \ddagger \\
{ }^{238} \mathrm{U}\end{array}$} & \multirow[b]{2}{*}{$\begin{array}{l} \pm 2 \sigma / \\
(\%)\end{array}$} & \multirow[b]{2}{*}{$\begin{array}{l}{ }^{207} \mathrm{~Pb} / \ddagger \\
{ }^{235} \mathrm{U}\end{array}$} & \multirow[b]{2}{*}{$\begin{array}{l} \pm 2 \sigma / \\
(\%)\end{array}$} & \multirow[b]{2}{*}{$\begin{array}{l}{ }^{207} \mathrm{~Pb} / \ddagger \\
{ }^{206} \mathrm{~Pb}\end{array}$} & \multirow[b]{2}{*}{$\begin{array}{l} \pm 2 \sigma / \\
(\%)\end{array}$} & \multirow[b]{2}{*}{ Rho§ } & \multicolumn{6}{|c|}{ Age (Ma) } & \multirow[b]{2}{*}{$\begin{array}{l}\text { Conce } \\
(\%)\end{array}$} \\
\hline & & & & & & & & & & & & & & $\begin{array}{l}206 \mathrm{~Pb} / \\
{ }^{238} \mathrm{U}\end{array}$ & $\pm 2 \sigma$ & $\begin{array}{l}{ }^{207} \mathrm{~Pb} / \\
{ }^{235} \mathrm{U}\end{array}$ & $\pm 2 \sigma$ & $\begin{array}{l}{ }^{207} \mathrm{~Pb} / \ddagger \\
{ }^{206} \mathrm{~Pb}\end{array}$ & $\pm 2 \sigma$ & \\
\hline $1 B$ & a-3 & 2494 & 71 & 5 & 0.12 & 8679 & 0.07886 & 2.4 & 0.6258 & 4.1 & 0.05756 & 3.3 & 0.59 & 489 & 11 & 493 & 16 & 513 & 73 & 95 \\
\hline $2 B$ & $a-6$ & 4675 & 137 & 11 & 0.27 & 10449 & 0.07999 & 2.0 & 0.6352 & 3.0 & 0.05759 & 2.2 & 0.67 & 496 & 10 & 499 & 12 & 514 & 49 & 96 \\
\hline $2 B$ & $a-7$ & 471 & 15 & 1 & 0.09 & 1667 & 0.07618 & 2.6 & 0.5933 & 7.5 & 0.05649 & 7.0 & 0.35 & 473 & 12 & 473 & 29 & 472 & 155 & 100 \\
\hline 3 & $\mathrm{a}-10$ & 433 & 13 & 1 & 0.09 & 1520 & 0.07826 & 2.5 & 0.6145 & 6.8 & 0.05695 & 6.3 & 0.37 & 486 & 12 & 486 & 27 & 490 & 139 & 99 \\
\hline 3 & $\mathrm{a}-11$ & 390 & 12 & 1 & 0.09 & 1402 & 0.07457 & 2.9 & 0.5726 & 8.9 & 0.05569 & 8.4 & 0.33 & 464 & 13 & 460 & 33 & 440 & 187 & 105 \\
\hline 4 & $a-12$ & 1498 & 50 & 4 & 0.12 & 5283 & 0.07873 & 2.2 & 0.6137 & 4.8 & 0.05654 & 4.2 & 0.47 & 489 & 11 & 486 & 19 & 473 & 94 & 103 \\
\hline 5 & $a-14$ & 1974 & 62 & 5 & 0.13 & 6919 & 0.07912 & 2.8 & 0.6210 & 4.0 & 0.05692 & 2.9 & 0.69 & 491 & 13 & 490 & 16 & 489 & 65 & 100 \\
\hline 7 & $\mathrm{a}-18$ & 1707 & 52 & 4 & 0.08 & 1557 & 0.07889 & 2.5 & 0.6204 & 3.8 & 0.05704 & 2.9 & 0.66 & 490 & 12 & 490 & 15 & 493 & 64 & 99 \\
\hline 7 & $a-19$ & 332 & 26 & 2 & 0.17 & 580 & 0.07692 & 2.6 & 0.6054 & 8.0 & 0.05709 & 7.5 & 0.33 & 478 & 12 & 481 & 31 & 495 & 165 & 96 \\
\hline 8 & $a-22$ & 953 & 36 & 2 & 0.18 & 3325 & 0.06839 & 2.6 & 0.5412 & 5.8 & 0.05739 & 5.1 & 0.45 & 426 & 11 & 439 & 21 & 507 & 113 & 84 \\
\hline 11 & $a-26$ & 3140 & 117 & 9 & 0.02 & 2239 & 0.08043 & 2.0 & 0.6281 & 3.3 & 0.05663 & 2.6 & 0.62 & 499 & 10 & 495 & 13 & 477 & 57 & 104 \\
\hline 12 & $a-27$ & 1662 & 64 & 5 & 0.20 & 2646 & 0.07770 & 2.2 & 0.6124 & 4.6 & 0.05717 & 4.0 & 0.49 & 482 & 10 & 485 & 18 & 498 & 88 & 97 \\
\hline 13 & $a-28$ & 4546 & 171 & 13 & 0.08 & 1758 & 0.07795 & 2.1 & 0.6254 & 3.2 & 0.05819 & 2.4 & 0.65 & 484 & 10 & 493 & 13 & 537 & 53 & 90 \\
\hline 15 & $a-32$ & 3923 & 151 & 12 & 0.22 & 13642 & 0.07997 & 2.4 & 0.6319 & 3.3 & 0.05731 & 2.2 & 0.73 & 496 & 12 & 497 & 13 & 503 & 49 & 99 \\
\hline 16 & $a-33$ & 3670 & 140 & 11 & 0.12 & 12700 & 0.07986 & 2.8 & 0.6341 & 4.0 & 0.05759 & 2.9 & 0.70 & 495 & 13 & 499 & 16 & 514 & 63 & 96 \\
\hline 16 & $a-34$ & 1082 & 44 & 3 & 0.06 & 460 & 0.07622 & 2.7 & 0.6000 & 5.6 & 0.05709 & 4.9 & 0.48 & 474 & 12 & 477 & 21 & 495 & 108 & 96 \\
\hline 23 & a-44 & 2599 & 98 & 8 & 0.22 & 970 & 0.07767 & 3.0 & 0.6222 & 4.5 & 0.05810 & 3.3 & 0.67 & 482 & 14 & 491 & 18 & 533 & 73 & 90 \\
\hline 24 & $a-45$ & 2773 & 112 & 9 & 0.14 & 9506 & 0.07889 & 2.1 & 0.6310 & 3.6 & 0.05801 & 3.0 & 0.57 & 489 & 10 & 497 & 14 & 530 & 65 & 92 \\
\hline 24 & $a-46$ & 532 & 27 & 2 & 0.13 & 264 & 0.07544 & 3.1 & 0.6001 & 10.4 & 0.05769 & 9.9 & 0.30 & 469 & 14 & 477 & 40 & 518 & 217 & 90 \\
\hline 25 & $a-47$ & 3079 & 112 & 10 & 0.30 & 4281 & 0.08187 & 2.4 & 0.6290 & 5.8 & 0.05572 & 5.3 & 0.41 & 507 & 12 & 495 & 23 & 441 & 118 & 115 \\
\hline 30 & a- 55 & 3383 & 136 & 11 & 0.13 & 3200 & 0.08298 & 3.1 & 0.6640 & 3.7 & 0.05804 & 2.1 & 0.82 & 514 & 15 & 517 & 15 & 531 & 47 & 97 \\
\hline 34 & $b-6$ & 2910 & 119 & 9 & 0.13 & 2746 & 0.07944 & 2.6 & 0.6203 & 4.0 & 0.05664 & 3.0 & 0.65 & 493 & 12 & 490 & 15 & 477 & 67 & 103 \\
\hline 34 & $b-7$ & 1251 & 49 & 4 & 0.16 & 4333 & 0.07686 & 3.0 & 0.6158 & 5.3 & 0.05811 & 4.4 & 0.56 & 477 & 14 & 487 & 21 & 534 & 96 & 89 \\
\hline 35 & $\mathrm{~b}-8$ & 1389 & 65 & 5 & 0.11 & 4804 & 0.07760 & 2.2 & 0.6228 & 4.3 & 0.05821 & 3.7 & 0.51 & 482 & 10 & 492 & 17 & 538 & 80 & 90 \\
\hline 35 & b-9 & 435 & 18 & 1 & 0.11 & 1476 & 0.07393 & 3.6 & 0.6012 & 9.2 & 0.05898 & 8.4 & 0.39 & 460 & 16 & 478 & 36 & 566 & 184 & 81 \\
\hline 35 & $b-10$ & 382 & 17 & 1 & 0.11 & 596 & 0.07270 & 3.6 & 0.5756 & 8.3 & 0.05742 & 7.5 & 0.43 & 452 & 16 & 462 & 31 & 508 & 164 & 89 \\
\hline 39 & $b-19$ & 2353 & 108 & 8 & 0.19 & 1020 & 0.07728 & 2.1 & 0.6090 & 3.5 & 0.05715 & 2.8 & 0.60 & 480 & 10 & 483 & 14 & 497 & 62 & 96 \\
\hline $41 \mathrm{~A}$ & $b-22$ & 3841 & 169 & 13 & 0.17 & 13509 & 0.07771 & 2.2 & 0.6149 & 3.3 & 0.05740 & 2.4 & 0.67 & 482 & 10 & 487 & 13 & 507 & 54 & 95 \\
\hline $41 \mathrm{~A}$ & $b-23$ & 362 & 16 & 1 & 0.11 & 1260 & 0.07743 & 3.6 & 0.6170 & 7.8 & 0.05779 & 6.9 & 0.46 & 481 & 17 & 488 & 31 & 522 & 152 & 92 \\
\hline 42 & b-25 & 1347 & 60 & 5 & 0.17 & 4678 & 0.07889 & 2.0 & 0.6262 & 4.6 & 0.05757 & 4.1 & 0.44 & 489 & 10 & 494 & 18 & 514 & 90 & 95 \\
\hline 42 & b-26 & 350 & 16 & 1 & 0.09 & 288 & 0.07584 & 3.2 & 0.6031 & 7.4 & 0.05768 & 6.6 & 0.43 & 471 & 14 & 479 & 29 & 517 & 146 & 91 \\
\hline 42 & $b-27$ & 205 & 9 & 1 & 0.09 & 728 & 0.07297 & 2.9 & 0.5759 & 11.6 & 0.05724 & 11.2 & 0.25 & 454 & 13 & 462 & 44 & 501 & 247 & 91 \\
\hline 43 & b-28 & 3588 & 151 & 12 & 0.15 & 12352 & 0.07984 & 2.7 & 0.6449 & 3.5 & 0.05858 & 2.3 & 0.75 & 495 & 13 & 505 & 14 & 552 & 51 & 90 \\
\hline 45 & $b-32$ & 2008 & 91 & 7 & 0.16 & 3412 & 0.08212 & 2.3 & 0.6399 & 4.0 & 0.05651 & 3.3 & 0.57 & 509 & 11 & 502 & 16 & 473 & 73 & 108 \\
\hline 46 & $b-33$ & 4652 & 196 & 17 & 0.21 & 4146 & 0.08309 & 2.5 & 0.6751 & 3.5 & 0.05893 & 2.5 & 0.71 & 515 & 12 & 524 & 15 & 564 & 54 & 91 \\
\hline 47 & b-34 & 1876 & 85 & 7 & 0.12 & 1120 & 0.07941 & 2.3 & 0.6293 & 4.1 & 0.05747 & 3.5 & 0.54 & 493 & 11 & 496 & 16 & 510 & 76 & 97 \\
\hline
\end{tabular}


Table 1 Continued

\begin{tabular}{|c|c|c|c|c|c|c|c|c|c|c|c|c|c|c|c|c|c|c|c|c|}
\hline \multirow[b]{2}{*}{ Grain } & \multirow[b]{2}{*}{ L-No. } & \multirow[b]{2}{*}{$\begin{array}{l}{ }^{207} \mathrm{~Pb}^{*} \\
\text { (cps) }\end{array}$} & \multirow[b]{2}{*}{$\begin{array}{l}\text { Uf } \\
\text { (ppm) }\end{array}$} & \multirow[b]{2}{*}{$\begin{array}{l}\mathrm{Pb} \dagger \\
(\mathrm{ppm})\end{array}$} & \multirow[b]{2}{*}{$\begin{array}{l}\text { Th/† } \\
U\end{array}$} & \multirow[b]{2}{*}{$\begin{array}{l}{ }^{206} \mathrm{~Pb} / \\
{ }^{204} \mathrm{~Pb}\end{array}$} & \multirow[b]{2}{*}{$\begin{array}{l}{ }^{206} \mathrm{~Pb} / \ddagger \\
{ }^{238} \mathrm{U}\end{array}$} & \multirow[b]{2}{*}{$\begin{array}{l} \pm 2 \sigma / \\
(\%)\end{array}$} & \multirow[b]{2}{*}{$\begin{array}{l}{ }^{207} \mathrm{~Pb} / \hbar \\
{ }^{235} \mathrm{U}\end{array}$} & \multirow[b]{2}{*}{$\begin{array}{l} \pm 2 \sigma / \\
(\%)\end{array}$} & \multirow[b]{2}{*}{$\begin{array}{l}{ }^{207} \mathrm{~Pb} / \hbar \\
{ }^{206} \mathrm{~Pb}\end{array}$} & \multirow[b]{2}{*}{$\begin{array}{l} \pm 2 \sigma / \\
(\%)\end{array}$} & \multirow[b]{2}{*}{ Rho§ } & \multicolumn{6}{|c|}{ Age (Ma) } & \multirow[b]{2}{*}{$\begin{array}{l}\text { Conc } \\
(\%)\end{array}$} \\
\hline & & & & & & & & & & & & & & $\begin{array}{l}{ }^{206} \mathrm{~Pb} / \\
{ }^{238} \mathrm{U}\end{array}$ & $\pm 2 \sigma$ & $\begin{array}{l}{ }^{207} \mathrm{~Pb} / \\
{ }^{235} \mathrm{U}\end{array}$ & $\pm 2 \sigma$ & $\begin{array}{l}{ }^{207} \mathrm{~Pb} / \$ \\
{ }^{206} \mathrm{~Pb}\end{array}$ & $\pm 2 \sigma$ & \\
\hline 49 & $b-38$ & 775 & 35 & 3 & 0.25 & 2718 & 0.08077 & 3.5 & 0.6398 & 6.9 & 0.05745 & 5.9 & 0.51 & 501 & 17 & 502 & 28 & 509 & 130 & 98 \\
\hline 50 & $b-39$ & 665 & 29 & 2 & 0.20 & 491 & 0.08163 & 2.7 & 0.6655 & 6.5 & 0.05913 & 5.9 & 0.41 & 506 & 13 & 518 & 27 & 572 & 129 & 88 \\
\hline 52 & $b-41$ & 1845 & 76 & 6 & 0.06 & 448 & 0.08024 & 3.2 & 0.6706 & 5.6 & 0.06061 & 4.7 & 0.56 & 498 & 15 & 521 & 23 & 626 & 100 & 80 \\
\hline 52 & $b-42$ & 415 & 20 & 1 & 0.06 & 1504 & 0.07730 & 3.3 & 0.5970 & 8.9 & 0.05601 & 8.3 & 0.37 & 480 & 15 & 475 & 34 & 453 & 184 & 106 \\
\hline 53 & b- 44 & 1283 & 61 & 5 & 0.11 & 4489 & 0.08086 & 2.6 & 0.6433 & 5.3 & 0.05770 & 4.7 & 0.49 & 501 & 13 & 504 & 21 & 518 & 103 & 97 \\
\hline 54 & b46 & 1243 & 54 & 4 & 0.23 & 782 & 0.08126 & 2.3 & 0.6585 & 4.6 & 0.05878 & 4.0 & 0.51 & 504 & 11 & 514 & 19 & 559 & 86 & 90 \\
\hline 55 & b48 & 1760 & 77 & 7 & 0.31 & 1325 & 0.08100 & 2.4 & 0.6384 & 4.3 & 0.05716 & 3.6 & 0.56 & 502 & 12 & 501 & 17 & 498 & 79 & 101 \\
\hline 58 & b51 & 1720 & 78 & 6 & 0.07 & 3086 & 0.08091 & 2.4 & 0.6470 & 4.1 & 0.05799 & 3.3 & 0.59 & 502 & 12 & 507 & 16 & 529 & 72 & 95 \\
\hline 59 & b53 & 2501 & 117 & 10 & 0.20 & 2938 & 0.08268 & 2.5 & 0.6619 & 4.2 & 0.05806 & 3.3 & 0.59 & 512 & 12 & 516 & 17 & 532 & 73 & 96 \\
\hline 62 & b54 & 1873 & 87 & 7 & 0.09 & 1068 & 0.08066 & 2.3 & 0.6375 & 4.4 & 0.05732 & 3.8 & 0.51 & 500 & 11 & 501 & 17 & 504 & 83 & 99 \\
\hline 62 & b55 & 337 & 18 & 1 & 0.08 & 1194 & 0.07910 & 2.3 & 0.6207 & 9.7 & 0.05691 & 9.5 & 0.23 & 491 & 11 & 490 & 39 & 488 & 209 & 101 \\
\hline 63 & $c-1$ & 4247 & 188 & 16 & 0.27 & 5955 & 0.08017 & 1.4 & 0.6448 & 3.2 & 0.05833 & 2.8 & 0.45 & 497 & 7 & 505 & 13 & 542 & 62 & 92 \\
\hline 64 & $c-2$ & 1672 & 74 & 6 & 0.24 & 5791 & 0.08034 & 1.6 & 0.6500 & 3.9 & 0.05868 & 3.5 & 0.41 & 498 & 8 & 508 & 16 & 555 & 77 & 90 \\
\hline 64 & $c-3$ & 780 & 36 & 3 & 0.01 & 2767 & 0.07786 & 3.1 & 0.6136 & 5.8 & 0.05716 & 5.0 & 0.52 & 483 & 14 & 486 & 23 & 498 & 109 & 97 \\
\hline 66 & $c-4$ & 3001 & 137 & 11 & 0.14 & 10476 & 0.08180 & 1.8 & 0.6556 & 3.4 & 0.05812 & 2.9 & 0.51 & 507 & 9 & 512 & 14 & 534 & 64 & 95 \\
\hline 68 & $c-7$ & 3701 & 171 & 14 & 0.23 & 1287 & 0.08025 & 1.5 & 0.6378 & 3.0 & 0.05764 & 2.7 & 0.49 & 498 & 7 & 501 & 12 & 516 & 58 & 96 \\
\hline 70 & $c-8$ & 2511 & 116 & 9 & 0.20 & 8707 & 0.07990 & 2.0 & 0.6410 & 3.8 & 0.05818 & 3.2 & 0.53 & 496 & 10 & 503 & 15 & 537 & 70 & 92 \\
\hline 70 & $c-9$ & 446 & 20 & 1 & 0.10 & 1506 & 0.07732 & 2.9 & 0.6438 & 7.4 & 0.06039 & 6.9 & 0.38 & 480 & 13 & 505 & 30 & 618 & 149 & 78 \\
\hline 72 & $c-10$ & 2819 & 137 & 11 & 0.21 & 9764 & 0.08026 & 1.5 & 0.6475 & 3.3 & 0.05851 & 2.9 & 0.45 & 498 & 7 & 507 & 13 & 549 & 64 & 91 \\
\hline 74 & $c-12$ & 3300 & 158 & 12 & 0.07 & 11539 & 0.08028 & 1.9 & 0.6426 & 3.5 & 0.05806 & 3.0 & 0.54 & 498 & 9 & 504 & 14 & 532 & 65 & 94 \\
\hline 76 & $c-14$ & 3389 & 177 & 14 & 0.10 & 11813 & 0.08083 & 1.5 & 0.6500 & 3.3 & 0.05832 & 2.9 & 0.46 & 501 & 7 & 508 & 13 & 542 & 64 & 92 \\
\hline 76 & $c-15$ & 330 & 17 & 1 & 0.07 & 1138 & 0.07888 & 2.9 & 0.6399 & 7.7 & 0.05883 & 7.1 & 0.38 & 489 & 14 & 502 & 31 & 561 & 155 & 87 \\
\hline 83 & $c-20$ & 375 & 22 & 2 & 0.12 & 458 & 0.07785 & 3.8 & 0.6279 & 7.3 & 0.05849 & 6.3 & 0.51 & 483 & 18 & 495 & 29 & 548 & 138 & 88 \\
\hline 87 & $c-22$ & 695 & 41 & 3 & 0.14 & 2469 & 0.08137 & 2.6 & 0.6430 & 5.7 & 0.05731 & 5.0 & 0.46 & 504 & 13 & 504 & 23 & 503 & 111 & 100 \\
\hline 88 & $c-24$ & 2171 & 127 & 11 & 0.26 & 7651 & 0.08155 & 3.3 & 0.6483 & 4.4 & 0.05766 & 2.9 & 0.75 & 505 & 16 & 507 & 18 & 517 & 64 & 98 \\
\hline 91 & $c-26$ & 2051 & 129 & 10 & 0.21 & 7269 & 0.07888 & 2.0 & 0.6220 & 4.2 & 0.05718 & 3.6 & 0.49 & 489 & 10 & 491 & 16 & 499 & 80 & 98 \\
\hline 99 & $c-31$ & 686 & 45 & 4 & 0.17 & 2405 & 0.08207 & 2.6 & 0.6521 & 6.7 & 0.05762 & 6.2 & 0.39 & 508 & 13 & 510 & 27 & 516 & 136 & 99 \\
\hline 99 & $c-32$ & 245 & 19 & 1 & 0.07 & 880 & 0.07539 & 3.7 & 0.5922 & 11.7 & 0.05697 & 11.1 & 0.32 & 469 & 17 & 472 & 45 & 490 & 245 & 96 \\
\hline 100 & $c-33$ & 2207 & 152 & 12 & 0.11 & 1126 & 0.08172 & 1.8 & 0.6510 & 3.7 & 0.05777 & 3.3 & 0.49 & 506 & 9 & 509 & 15 & 521 & 72 & 97 \\
\hline 102 & $c-37$ & 1962 & 146 & 12 & 0.21 & 1535 & 0.07995 & 2.4 & 0.6437 & 3.9 & 0.05840 & 3.1 & 0.60 & 496 & 11 & 505 & 16 & 545 & 69 & 91 \\
\hline 102 & $c-38$ & 201 & 15 & 1 & 0.14 & 732 & 0.07476 & 3.3 & 0.6041 & 12.9 & 0.05861 & 12.4 & 0.25 & 465 & 15 & 480 & 50 & 553 & 271 & 84 \\
\hline 105 & $c-39$ & 1613 & 127 & 10 & 0.11 & 5542 & 0.08029 & 1.9 & 0.6417 & 3.8 & 0.05797 & 3.3 & 0.49 & 498 & 9 & 503 & 15 & 528 & 73 & 94 \\
\hline 106 & $c-40$ & 453 & 42 & 3 & 0.15 & 1640 & 0.07582 & 2.0 & 0.5845 & 7.5 & 0.05591 & 7.2 & 0.27 & 471 & 9 & 467 & 28 & 449 & 161 & 105 \\
\hline 115 & $c-45$ & 723 & 66 & 5 & 0.07 & 2549 & 0.08044 & 3.4 & 0.6353 & 6.7 & 0.05728 & 5.8 & 0.51 & 499 & 16 & 499 & 27 & 502 & 127 & 99 \\
\hline 115 & $c-46$ & 124 & 12 & 1 & 0.07 & 462 & 0.07667 & 5.0 & 0.5832 & 12.4 & 0.05517 & 11.4 & 0.40 & 476 & 23 & 467 & 47 & 419 & 254 & 114 \\
\hline $116 \mathrm{~A}$ & $c-47$ & 4282 & 385 & 31 & 0.16 & 14973 & 0.08009 & 2.4 & 0.6410 & 3.3 & 0.05804 & 2.3 & 0.72 & 497 & 11 & 503 & 13 & 531 & 50 & 93 \\
\hline $116 \mathrm{~A}$ & $c-48$ & 1001 & 102 & 7 & 0.07 & 3642 & 0.07684 & 2.3 & 0.5913 & 5.0 & 0.05581 & 4.5 & 0.45 & 477 & 10 & 472 & 19 & 445 & 99 & 107 \\
\hline 1168 & $c-49$ & 1715 & 178 & 13 & 0.05 & 6241 & 0.07932 & 1.4 & 0.6117 & 4.0 & 0.05593 & 3.7 & 0.36 & 492 & 7 & 485 & 15 & 449 & 82 & 109 \\
\hline 1168 & $c-50$ & 449 & 48 & 4 & 0.20 & 1585 & 0.07618 & 2.6 & 0.6049 & 7.2 & 0.05759 & 6.8 & 0.35 & 473 & 12 & 480 & 28 & 514 & 149 & 92 \\
\hline 117 & $c-53$ & 781 & 87 & 7 & 0.13 & 2769 & 0.07771 & 2.8 & 0.6106 & 6.0 & 0.05699 & 5.3 & 0.46 & 482 & 13 & 484 & 23 & 491 & 117 & 98 \\
\hline $118 \mathrm{~A}$ & $c-54$ & 2854 & 305 & 23 & 0.09 & 9986 & 0.07821 & 2.1 & 0.6218 & 3.7 & 0.05767 & 3.1 & 0.56 & 485 & 10 & 491 & 15 & 517 & 67 & 94 \\
\hline
\end{tabular}


Table 1 Continued.

\begin{tabular}{|c|c|c|c|c|c|c|c|c|c|c|c|c|c|c|c|c|c|c|c|c|}
\hline \multirow[b]{2}{*}{ Grain } & \multirow[b]{2}{*}{ L-No. } & \multirow[b]{2}{*}{$\begin{array}{l}{ }^{207} \mathrm{~Pb}^{*} \\
\text { (cps) }\end{array}$} & \multirow[b]{2}{*}{$\begin{array}{l}\text { Uf } \\
(\mathrm{ppm})\end{array}$} & \multirow[b]{2}{*}{$\begin{array}{l}\mathrm{Pb} \dagger \\
(\mathrm{ppm})\end{array}$} & \multirow[b]{2}{*}{$\begin{array}{l}\text { Th/† } \\
U\end{array}$} & \multirow[b]{2}{*}{$\begin{array}{l}{ }^{206} \mathrm{~Pb} / \\
{ }^{204} \mathrm{~Pb}\end{array}$} & \multirow[b]{2}{*}{$\begin{array}{l}{ }^{206} \mathrm{~Pb} / \ddagger \\
{ }^{238} \mathrm{U}\end{array}$} & \multirow[b]{2}{*}{$\begin{array}{l} \pm 2 \sigma / \\
(\%)\end{array}$} & \multirow[b]{2}{*}{$\begin{array}{l}{ }^{207} \mathrm{~Pb} / \$ \\
{ }^{235} \mathrm{U}\end{array}$} & \multirow[b]{2}{*}{$\begin{array}{l} \pm 2 \sigma / \\
(\%)\end{array}$} & \multirow[b]{2}{*}{$\begin{array}{l}{ }^{207} \mathrm{~Pb} / \neq \\
{ }^{206} \mathrm{~Pb}\end{array}$} & \multirow[b]{2}{*}{$\begin{array}{l} \pm 2 \sigma / \\
(\%)\end{array}$} & \multirow[b]{2}{*}{ Rho§ } & \multicolumn{6}{|c|}{ Age (Ma) } & \multirow[b]{2}{*}{$\begin{array}{l}\text { Conc } 9 \\
(\%)\end{array}$} \\
\hline & & & & & & & & & & & & & & $\begin{array}{l}{ }^{206} \mathrm{~Pb} / \\
{ }^{238} \mathrm{U}\end{array}$ & $\pm 2 \sigma$ & $\begin{array}{l}{ }^{207} \mathrm{~Pb} / \\
{ }^{235} \mathrm{U}\end{array}$ & $\pm 2 \sigma$ & $\begin{array}{l}{ }^{207} \mathrm{~Pb} / \neq \\
{ }^{206} \mathrm{~Pb}\end{array}$ & $\pm 2 \sigma$ & \\
\hline $118 \mathrm{~A}$ & $c-55$ & 353 & 38 & 3 & 0.07 & 1222 & 0.07470 & 4.2 & 0.6052 & 8.0 & 0.05876 & 6.8 & 0.52 & 464 & 19 & 481 & 31 & 558 & 148 & 83 \\
\hline $118 B$ & $\mathrm{~d}-1$ & 2073 & 91 & 7 & 0.13 & 7190 & 0.07926 & 2.2 & 0.6182 & 4.1 & 0.05658 & 3.5 & 0.53 & 492 & 10 & 489 & 16 & 475 & 78 & 104 \\
\hline 119 & $d-3$ & 2971 & 132 & 11 & 0.23 & 10303 & 0.07843 & 2.0 & 0.6135 & 3.4 & 0.05672 & 2.8 & 0.59 & 487 & 9 & 486 & 13 & 481 & 61 & 101 \\
\hline 119 & $\mathrm{~d}-4$ & 545 & 23 & 2 & 0.13 & 1150 & 0.07632 & 2.8 & 0.6269 & 9.6 & 0.05957 & 9.2 & 0.29 & 474 & 13 & 494 & 38 & 588 & 200 & 81 \\
\hline 121 & $d-5$ & 717 & 31 & 2 & 0.07 & 1111 & 0.07770 & 2.8 & 0.6136 & 6.1 & 0.05728 & 5.4 & 0.45 & 482 & 13 & 486 & 24 & 502 & 119 & 96 \\
\hline 121 & $d-6$ & 5402 & 236 & 19 & 0.23 & 18769 & 0.08045 & 2.2 & 0.6290 & 2.9 & 0.05670 & 1.9 & 0.76 & 499 & 11 & 495 & 12 & 480 & 42 & 104 \\
\hline 125 & $d-10$ & 6127 & 267 & 21 & 0.13 & 21185 & 0.08107 & 2.2 & 0.6375 & 2.9 & 0.05703 & 1.8 & 0.77 & 503 & 11 & 501 & 11 & 493 & 40 & 102 \\
\hline 125 & $d-11$ & 3086 & 140 & 11 & 0.10 & 3387 & 0.07852 & 2.2 & 0.6108 & 3.7 & 0.05642 & 2.9 & 0.60 & 487 & 10 & 484 & 14 & 469 & 65 & 104 \\
\hline $126 \mathrm{~A}$ & $d-12$ & 3347 & 151 & 11 & 0.04 & 3519 & 0.07948 & 2.4 & 0.6373 & 3.8 & 0.05815 & 3.0 & 0.62 & 493 & 11 & 501 & 15 & 535 & 65 & 92 \\
\hline 1268 & $d-14$ & 2485 & 112 & 9 & 0.11 & 1513 & 0.07862 & 2.6 & 0.6337 & 4.1 & 0.05846 & 3.2 & 0.62 & 488 & 12 & 498 & 16 & 547 & 71 & 89 \\
\hline 127 & $d-15$ & 2694 & 118 & 9 & 0.17 & 9067 & 0.07865 & 2.4 & 0.6347 & 3.6 & 0.05853 & 2.7 & 0.66 & 488 & 11 & 499 & 14 & 550 & 58 & 89 \\
\hline 128 & $d-17$ & 3153 & 139 & 11 & 0.21 & 2549 & 0.07881 & 1.8 & 0.6334 & 3.1 & 0.05830 & 2.5 & 0.59 & 489 & 9 & 498 & 12 & 541 & 55 & 90 \\
\hline 130 & $d-20$ & 2823 & 130 & 10 & 0.22 & 1869 & 0.07869 & 2.0 & 0.6102 & 3.3 & 0.05624 & 2.5 & 0.63 & 488 & 10 & 484 & 13 & 462 & 56 & 106 \\
\hline 134 & $d-25$ & 2709 & 124 & 9 & 0.08 & 9236 & 0.07819 & 2.2 & 0.6239 & 4.0 & 0.05787 & 3.4 & 0.55 & 485 & 10 & 492 & 16 & 525 & 74 & 92 \\
\hline 135 & $d-27$ & 1977 & 91 & 7 & 0.23 & 6549 & 0.07992 & 2.2 & 0.6554 & 3.8 & 0.05948 & 3.1 & 0.59 & 496 & 11 & 512 & 15 & 585 & 67 & 85 \\
\hline 135 & $d-28$ & 183 & 9 & 1 & 0.09 & 646 & 0.07479 & 3.8 & 0.5834 & 11.6 & 0.05657 & 11.0 & 0.32 & 465 & 17 & 467 & 44 & 475 & 243 & 98 \\
\hline 140 & $d-33$ & 1765 & 82 & 6 & 0.09 & 5955 & 0.08232 & 2.5 & 0.6615 & 4.4 & 0.05827 & 3.7 & 0.56 & 510 & 12 & 516 & 18 & 540 & 80 & 94 \\
\hline
\end{tabular}

Diameter of laser spot $=20 \mathrm{pm}$; depth of crater ca. $15 \mu \mathrm{m}$.

*Within run background-corrected mean ${ }^{207} \mathrm{~Pb}$ signal in counts per second.

$\mp U$ and $\mathrm{Pb}$ content and Th/ $\mathrm{U}$ ratio were calculated relative to $\mathrm{GJ}-1$ reference (LA-ICP-MS values).

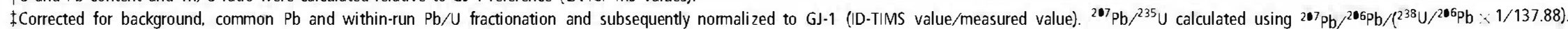
Uncertainties propagated following Gerdes and Zeh $(2006,2009)$.

$\S$ Rho is the error correlation of the ${ }^{206} \mathrm{~Pb} /{ }^{238} \mathrm{U}$ and ${ }^{207} \mathrm{~Pb} /{ }^{235} \mathrm{U}$ errors.

I|Percent concordance $={ }^{206} \mathrm{~Pb} /{ }^{238} \mathrm{U}$ age $/{ }^{207} \mathrm{~Pb} /{ }^{206} \mathrm{~Pb}$ age $\times 100$. 
Table 2 LA-MC-ICPMS Lu-Hf isotope data of zircon from the sample of Bazar amphibolite

\begin{tabular}{|c|c|c|c|c|c|c|c|c|c|c|c|c|c|c|c|}
\hline L-No. & ${ }^{176} \mathrm{Yb} /{ }^{177} \mathrm{Hf}^{*}$ & $\pm 2 \sigma$ & ${ }^{176} \mathrm{LW}^{177} \mathrm{Hf}^{*}$ & $\pm 2 \sigma$ & ${ }^{178} \mathrm{Hf} /{ }^{177} \mathrm{Hf}$ & ${ }^{180} \mathrm{Hf} /{ }^{177} \mathrm{Hf}$ & $\operatorname{Sig}_{\mathrm{Hf}^{\dagger}}(\mathrm{M})$ & ${ }^{176} \mathrm{Hf} /{ }^{177} \mathrm{Hf}$ & $\pm 2 \sigma \ddagger$ & ${ }^{176} \mathrm{Hf} / /^{177} \mathrm{Hf}_{(t)}$ & $\varepsilon H f(t) \S$ & $\pm 2 \sigma$ & $T_{D M 2}$ I (Ga) & $\mathrm{Age}^{* *}(\mathrm{Ma})$ & $\pm 2 \sigma$ \\
\hline a-3 & 0.0194 & 16 & 0.00074 & 5 & 1.46717 & 1.88676 & 6 & 0.282845 & 29 & 0.282838 & 12.8 & 0.7 & 0.61 & 489 & 11 \\
\hline$a \cdot 6$ & 0.0130 & 11 & 0.00049 & 3 & 1.46715 & 1.88670 & 8 & 0.282865 & 27 & 0.282860 & 13.7 & 0.6 & 0.56 & 496 & 10 \\
\hline$a-7$ & 0.0071 & 6 & 0.00027 & 2 & 1.46720 & 1.88669 & 6 & 0.282864 & 24 & 0.282861 & 13.2 & 0.5 & 0.57 & 473 & 12 \\
\hline$a-10$ & 0.0070 & 6 & 0.00028 & 2 & 1.46717 & 1.88681 & 7 & 0.282854 & 29 & 0.282852 & 13.2 & 0.7 & 0.58 & 486 & 12 \\
\hline$a \cdot 12$ & 0.0125 & 11 & 0.00045 & 3 & 1.46715 & 1.88671 & 7 & 0.282832 & 26 & 0.282828 & 12.4 & 0.6 & 0.63 & 489 & 11 \\
\hline$a-14$ & 0.0126 & 11 & 0.00045 & 3 & 1.46716 & 1.88656 & 8 & 0.282861 & 23 & 0.282857 & 13.5 & 0.5 & 0.57 & 491 & 13 \\
\hline$a-18$ & 0.0080 & 7 & 0.00031 & 2 & 1.46715 & 1.88669 & 6 & 0.282855 & 29 & 0.282852 & 13.3 & 0.7 & 0.58 & 490 & 12 \\
\hline$a-22$ & 0.0086 & 8 & 0.00034 & 2 & 1.46707 & 1.88656 & 7 & 0.282850 & 26 & 0.282847 & 11.7 & 0.6 & 0.62 & 426 & 11 \\
\hline$a-26$ & 0.0178 & 14 & 0.00083 & 5 & 1.46719 & 1.88670 & 6 & 0.282859 & 30 & 0.282851 & 13.5 & 0.7 & 0.58 & 499 & 10 \\
\hline$a-27$ & 0.0129 & 11 & 0.00049 & 3 & 1.46721 & 1.88663 & 6 & 0.282869 & 26 & 0.282864 & 13.6 & 0.6 & 0.56 & 482 & 10 \\
\hline$a-28$ & 0.0114 & 13 & 0.00041 & 4 & 1.46724 & 1.88689 & 8 & 0.282812 & 28 & 0.282808 & 11.6 & 0.7 & 0.67 & 484 & 10 \\
\hline a-32 & 0.0142 & 11 & 0.00053 & 3 & 1.46717 & 1.88662 & 7 & 0.282857 & 25 & 0.282852 & 13.4 & 0.5 & 0.58 & 496 & 12 \\
\hline$a-33$ & 0.0151 & 12 & 0.00054 & 4 & 1.46722 & 1.88690 & 7 & 0.282836 & 24 & 0.282831 & 12.7 & 0.5 & 0.62 & 495 & 13 \\
\hline$a-34$ & 0.0087 & 7 & 0.00033 & 2 & 1.46727 & 1.88687 & 7 & 0.282846 & 24 & 0.282843 & 12.6 & 0.5 & 0.60 & 474 & 12 \\
\hline a- 42 & 0.0112 & 9 & 0.00041 & 3 & 1.46722 & 1.88688 & 8 & 0.282818 & 23 & 0.282814 & 11.9 & 0.5 & 0.66 & 485 & 12 \\
\hline$a-43$ & 0.0161 & 13 & 0.00062 & 4 & 1.46717 & 1.88649 & 7 & 0.282852 & 32 & 0.282846 & 12.8 & 0.8 & 0.60 & 478 & 14 \\
\hline a-44 & 0.0168 & 14 & 0.00064 & 4 & 1.46716 & 1.88667 & 7 & 0.282833 & 24 & 0.282827 & 12.3 & 0.5 & 0.63 & 482 & 14 \\
\hline$a-45$ & 0.0129 & 11 & 0.00049 & 3 & 1.46713 & 1.88663 & 7 & 0.282828 & 27 & 0.282824 & 12.3 & 0.6 & 0.63 & 489 & 10 \\
\hline$a-47$ & 0.0211 & 17 & 0.00077 & 5 & 1.46712 & 1.88671 & 7 & 0.282838 & 29 & 0.282830 & 12.9 & 0.7 & 0.61 & 507 & 12 \\
\hline a- 55 & 0.0128 & 10 & 0.00048 & 3 & 1.46707 & 1.88652 & 6 & 0.282875 & 25 & 0.282871 & 14.5 & 0.6 & 0.53 & 514 & 15 \\
\hline b- 6 & 0.0367 & 32 & 0.00082 & 5 & 1.46727 & 1.88709 & 8 & 0.282863 & 34 & 0.282855 & 13.5 & 1.2 & 0.57 & 493 & 12 \\
\hline b-8 & 0.0121 & 12 & 0.00031 & 2 & 1.46728 & 1.88697 & 4 & 0.282901 & 41 & 0.282898 & 14.7 & 1.5 & 0.49 & 482 & 10 \\
\hline b-9 9 & 0.0146 & 12 & 0.00042 & 3 & 1.46727 & 1.88665 & 4 & 0.282870 & 52 & 0.282866 & 13.1 & 1.8 & 0.56 & 460 & 16 \\
\hline b-19 & 0.0214 & 17 & 0.00053 & 3 & 1.46723 & 1.88671 & 10 & 0.282865 & 26 & 0.282860 & 13.4 & 0.9 & 0.57 & 480 & 10 \\
\hline b-22 & 0.0281 & 23 & 0.00071 & 4 & 1.46713 & 1.88673 & 7 & 0.282873 & 31 & 0.282866 & 13.6 & 1.1 & 0.55 & 482 & 10 \\
\hline b. 23 & 0.0095 & 8 & 0.00026 & 2 & 1.46723 & 1.88679 & 11 & 0.282867 & 32 & 0.282865 & 13.5 & 1.1 & 0.56 & 481 & 17 \\
\hline b- 25 & 0.0228 & 20 & 0.00055 & 3 & 1.46721 & 1.88668 & 8 & 0.282858 & 26 & 0.282853 & 13.3 & 0.9 & 0.58 & 489 & 10 \\
\hline b- 26 & 0.0129 & 13 & 0.00033 & 3 & 1.46725 & 1.88654 & 9 & 0.282866 & 32 & 0.282863 & 13.3 & 1.1 & 0.56 & 471 & 14 \\
\hline b-27 & 0.0120 & 11 & 0.00031 & 2 & 1.46721 & 1.88678 & 9 & 0.282848 & 29 & 0.282846 & 12.3 & 1.0 & 0.61 & 454 & 13 \\
\hline b-28 & 0.0172 & 14 & 0.00039 & 2 & 1.46720 & 1.88704 & 7 & 0.282836 & 29 & 0.282832 & 12.7 & 1.0 & 0.62 & 495 & 13 \\
\hline b-32 & 0.0232 & 19 & 0.00058 & 3 & 1.46720 & 1.88686 & 9 & 0.282833 & 23 & 0.282828 & 12.9 & 0.8 & 0.62 & 509 & 11 \\
\hline b-34 & 0.0201 & 16 & 0.00051 & 3 & 1.46719 & 1.88689 & 8 & 0.282881 & 40 & 0.282877 & 14.2 & 1.4 & 0.53 & 493 & 11 \\
\hline b. 35 & 0.0090 & 8 & 0.00027 & $z$ & 1.46726 & 1.88663 & 12 & 0.282861 & 23 & 0.282859 & 13.2 & 0.8 & 0.57 & 473 & 16 \\
\hline b-37 & 0.0228 & 19 & 0.00053 & 3 & 1.46724 & 1.88662 & 7 & 0.282895 & 29 & 0.282890 & 14.9 & 1.0 & 0.50 & 502 & 12 \\
\hline b-38 & 0.0204 & 18 & 0.00048 & 3 & 1.46722 & 1.88644 & 8 & 0.282862 & 30 & 0.282857 & 13.7 & 1.0 & 0.56 & 501 & 17 \\
\hline b. 39 & 0.0315 & 25 & 0.00074 & 4 & 1.46722 & 1.88690 & 9 & 0.282819 & 28 & 0.282812 & 12.3 & 1.0 & 0.65 & 506 & 13 \\
\hline b-41 & 0.0097 & 9 & 0.00031 & 2 & 1.46722 & 1.88651 & 13 & 0.282877 & 21 & 0.282874 & 14.2 & 0.7 & 0.53 & 498 & 15 \\
\hline b-42 & 0.0090 & 7 & 0.00025 & 1 & 1.46720 & 1.88705 & 12 & 0.282810 & 26 & 0.282808 & 11.5 & 0.9 & 0.67 & 480 & 15 \\
\hline b-44 & 0.0342 & 28 & 0.00080 & 5 & 1.46719 & 1.88688 & 9 & 0.282855 & 24 & 0.282848 & 13.4 & 0.8 & 0.58 & 501 & 13 \\
\hline b- 46 & 0.0241 & 20 & 0.00056 & 3 & 1.46721 & 1.88653 & 8 & 0.282880 & 24 & 0.282874 & 14.4 & 0.9 & 0.53 & 504 & 11 \\
\hline b- 48 & 0.0289 & 23 & 0.00069 & 4 & 1.46719 & 1.88689 & 8 & 0.282859 & 34 & 0.282852 & 13.6 & 1.2 & 0.57 & 502 & 12 \\
\hline b-51 & 0.0248 & 20 & 0.00060 & 4 & 1.46722 & 1.88671 & 9 & 0.282825 & 31 & 0.282819 & 12.4 & 1.1 & 0.64 & 502 & 12 \\
\hline b. 53 & 0.0188 & 15 & 0.00046 & 3 & 1.46723 & 1.88686 & 9 & 0.282851 & 30 & 0.282846 & 13.6 & 1.0 & 0.58 & 512 & 12 \\
\hline b-54 & 0.0095 & 8 & 0.00023 & 1 & 1.46724 & 1.88647 & 8 & 0.282838 & 31 & 0.282836 & 13.0 & 1.1 & 0.61 & 500 & 11 \\
\hline
\end{tabular}


Table 2 Continued

\begin{tabular}{|c|c|c|c|c|c|c|c|c|c|c|c|c|c|c|c|}
\hline L-No. & ${ }^{176} \mathrm{Yb} /{ }^{177} \mathrm{Hf}{ }^{*}$ & $\pm 2 \sigma$ & ${ }^{176} \mathrm{Lu} /{ }^{177} \mathrm{Hf}^{*}$ & $\pm 2 \sigma$ & ${ }^{178} \mathrm{Hf} /{ }^{177} \mathrm{Hf}$ & ${ }^{180} \mathrm{Hf} /{ }^{177} \mathrm{Hf}$ & $\operatorname{sig}_{\mathrm{H} f} f^{\dagger}(M)$ & ${ }^{176} \mathrm{Hf} /{ }^{177} \mathrm{Hf}$ & $\pm 2 \sigma \ddagger$ & ${ }^{176} \mathrm{Hf} /{ }^{177} \mathrm{Hf}_{(t)}$ & $\varepsilon H f(t) \S$ & $\pm 2 \sigma$ & $T_{D M_{2}}$ I (Ga) & Age $^{* *}(\mathrm{Ma})$ & $\pm 2 \sigma$ \\
\hline b- 55 & 0.0059 & 5 & 0.00018 & 1 & 1.46725 & 1.88622 & 11 & 0.282866 & 30 & 0.282865 & 13.8 & 1.1 & 0.55 & 491 & 11 \\
\hline$c-1$ & 0.0238 & 19 & 0.00070 & 4 & 1.46725 & 1.88675 & 12 & 0.282855 & 22 & 0.282849 & 13.3 & 0.8 & 0.58 & 497 & 7 \\
\hline$c-2$ & 0.0139 & 11 & 0.00043 & 3 & 1.46723 & 1.88649 & 4 & 0.282856 & 36 & 0.282852 & 13.5 & 1.3 & 0.57 & 498 & 8 \\
\hline$c-3$ & 0.0109 & 9 & 0.00036 & 2 & 1.46719 & 1.88674 & 5 & 0.282834 & 37 & 0.282830 & 12.4 & 1.3 & 0.62 & 483 & 14 \\
\hline$c-7$ & 0.0190 & 16 & 0.00058 & 4 & 1.46723 & 1.88667 & 5 & 0.282854 & 27 & 0.282849 & 13.4 & 0.9 & 0.58 & 498 & 7 \\
\hline$c-8$ & 0.0161 & 13 & 0.00052 & 3 & 1.46721 & 1.88660 & 5 & 0.282864 & 27 & 0.282859 & 13.7 & 0.9 & 0.56 & 496 & 10 \\
\hline$c-9$ & 0.0089 & 8 & 0.00034 & 2 & 1.46724 & 1.88656 & 4 & 0.282856 & 43 & 0.282853 & 13.1 & 1.5 & 0.58 & 480 & 13 \\
\hline$c-10$ & 0.0131 & 11 & 0.00043 & 3 & 1.46726 & 1.88661 & 5 & 0.282860 & 39 & 0.282856 & 13.6 & 1.4 & 0.57 & 498 & 7 \\
\hline$c-12$ & 0.0411 & 33 & 0.00135 & 8 & 1.46723 & 1.88676 & 6 & 0.282867 & 32 & 0.282854 & 13.5 & 1.1 & 0.57 & 498 & 9 \\
\hline c- 14 & 0.0164 & 14 & 0.00053 & 3 & 1.46720 & 1.88647 & 6 & 0.282842 & 25 & 0.282837 & 13.0 & 0.9 & 0.60 & 501 & 7 \\
\hline$c-15$ & 0.0074 & 6 & 0.00025 & 2 & 1.46723 & 1.88649 & 5 & 0.282866 & 28 & 0.282863 & 13.7 & 1.0 & 0.56 & 489 & 14 \\
\hline$c-22$ & 0.0099 & 8 & 0.00034 & 2 & 1.46722 & 1.88693 & 6 & 0.282867 & 32 & 0.282864 & 14.0 & 1.1 & 0.55 & 504 & 13 \\
\hline$c-24$ & 0.0226 & 18 & 0.00071 & 4 & 1.46721 & 1.88678 & 7 & 0.282867 & 30 & 0.282860 & 13.9 & 1.1 & 0.56 & 505 & 16 \\
\hline$c-26$ & 0.0191 & 15 & 0.00060 & 4 & 1.46727 & 1.88648 & 6 & 0.282889 & 29 & 0.282883 & 14.4 & 1.0 & 0.52 & 489 & 10 \\
\hline$c-31$ & 0.0137 & 15 & 0.00043 & 4 & 1.46722 & 1.88682 & 6 & 0.282829 & 34 & 0.282825 & 12.8 & 1.2 & 0.62 & 508 & 13 \\
\hline$c-32$ & 0.0066 & 5 & 0.00023 & 1 & 1.46725 & 1.88675 & 7 & 0.282855 & 26 & 0.282853 & 12.9 & 0.9 & 0.59 & 469 & 17 \\
\hline$c .33$ & 0.0153 & 13 & 0.00049 & 3 & 1.46723 & 1.88670 & 5 & 0.282836 & 35 & 0.282832 & 12.9 & 1.3 & 0.61 & 506 & 9 \\
\hline$c-37$ & 0.0190 & 17 & 0.00063 & 4 & 1.46721 & 1.88655 & 6 & 0.282859 & 31 & 0.282853 & 13.5 & 1.1 & 0.58 & 496 & 11 \\
\hline$c-38$ & 0.0067 & 6 & 0.00022 & 1 & 1.46726 & 1.88681 & 6 & 0.282810 & 29 & 0.282808 & 11.2 & 1.0 & 0.68 & 465 & 15 \\
\hline$c-39$ & 0.0115 & 9 & 0.00038 & 2 & 1.46730 & 1.88687 & 7 & 0.282821 & 30 & 0.282818 & 12.3 & 1.1 & 0.64 & 498 & 9 \\
\hline$c-40$ & 0.0092 & 8 & 0.00032 & 2 & 1.46727 & 1.88662 & 6 & 0.282862 & 31 & 0.282859 & 13.1 & 1.1 & 0.57 & 471 & 9 \\
\hline$c-45$ & 0.0190 & 15 & 0.00059 & 4 & 1.46727 & 1.88680 & 10 & 0.282830 & 26 & 0.282824 & 12.5 & 0.9 & 0.63 & 499 & 16 \\
\hline$c-46$ & 0.0095 & 9 & 0.00033 & 3 & 1.46721 & 1.88658 & 5 & 0.282870 & 30 & 0.282867 & 13.5 & 1.1 & 0.55 & 476 & 23 \\
\hline$c-47$ & 0.0236 & 22 & 0.00073 & 5 & 1.46725 & 1.88640 & 6 & 0.282870 & 30 & 0.282863 & 13.8 & 1.1 & 0.55 & 497 & 11 \\
\hline$c-48$ & 0.0112 & 9 & 0.00037 & 2 & 1.46719 & 1.88679 & 6 & 0.282850 & 29 & 0.282847 & 12.8 & 1.0 & 0.59 & 477 & 10 \\
\hline$c-49$ & 0.0128 & 13 & 0.00048 & 4 & 1.46728 & 1.88679 & 6 & 0.282866 & 35 & 0.282862 & 13.7 & 1.2 & 0.56 & 492 & 7 \\
\hline$c-50$ & 0.0140 & 11 & 0.00052 & 3 & 1.46727 & 1.88653 & 5 & 0.282869 & 34 & 0.282865 & 13.4 & 1.2 & 0.56 & 473 & 12 \\
\hline$c-54$ & 0.0258 & 22 & 0.00079 & 5 & 1.46720 & 1.88637 & 6 & 0.282901 & 33 & 0.282894 & 14.7 & 1.2 & 0.50 & 485 & 10 \\
\hline$c-55$ & 0.0067 & 6 & 0.00025 & 2 & 1.46722 & 1.88670 & 5 & 0.282852 & 34 & 0.282850 & 12.6 & 1.2 & 0.59 & 464 & 19 \\
\hline$d-1$ & 0.0120 & 10 & 0.00039 & 2 & 1.46720 & 1.88674 & 5 & 0.282839 & 32 & 0.282835 & 12.7 & 1.1 & 0.61 & 492 & 10 \\
\hline d-4 & 0.0087 & 7 & 0.00030 & 2 & 1.46728 & 1.88673 & 5 & 0.282818 & 27 & 0.282816 & 11.7 & 0.9 & 0.66 & 474 & 13 \\
\hline$d-5$ & 0.0065 & 5 & 0.00021 & 1 & 1.46730 & 1.88665 & 6 & 0.282849 & 27 & 0.282847 & 12.9 & 0.9 & 0.59 & 482 & 13 \\
\hline$d-20$ & 0.0104 & 9 & 0.00035 & 2 & 1.46724 & 1.88630 & 5 & 0.282871 & 40 & 0.282867 & 13.8 & 1.4 & 0.55 & 488 & 10 \\
\hline
\end{tabular}

${ }^{176} \mathrm{Vb} /{ }^{177} \mathrm{Hf}=\left({ }^{176} \mathrm{Yb} /{ }^{173} \mathrm{Yb}\right)$ true $\left.\times{ }^{173} \mathrm{Yb} /{ }^{177} \mathrm{Hf}\right)$ meas $\times(\mathrm{M} 173(\mathrm{Vb}) / \mathrm{M} 177(\mathrm{Hf}))^{z(\mathrm{H} f)}$. The ${ }^{176} \mathrm{LU} /{ }^{177} \mathrm{HF}$ were calculated in a similar way by using the ${ }^{175}$ Lu $/{ }^{177} \mathrm{Hf}$. Quoted uncertainties (absolute) relate to the last quoted figure. The effect of the inter-element fractionation on the Lu/Hf was estimated to be about $6 \%$ or less based on analyses of the GJ-1 and Plesovice zircons.

$\dagger$ †Mean Hf signal in volt.

IUncertainties are quadratic additions of the within-run error and the daily reproducibility of the 40ppb-JMC475 solution. Uncertainties for the GJ-1 are 2SD.

SInitial ${ }^{176} \mathrm{HF} /{ }^{177} \mathrm{Hf}$ and $\varepsilon \mathrm{Hf}$ calculated using the age determined by LA-ICP-MS dating (see last two rows).

"Two-stage model age in billion years using the measured ${ }^{176} \mathrm{Lu} /{ }^{177} \mathrm{Lu}$ of each spot (first stage $=$ age of zircon), a value of 0.0113 for the average continental crust (second stage), and a depleted mantle ${ }^{176} \mathrm{Lu} /{ }^{177} \mathrm{Lu}$ and ${ }^{176} \mathrm{Hf} /{ }^{177} \mathrm{Hf}$ of 0.0384 and 0.28325 , respectively (see Gerdes and Zeh, 2006 for details and references)

** LA-ICP-MS age and error (see Table 1 for more details). 
Seventy five per cent of the acquired $\mathrm{U}-\mathrm{Pb}$ analyses are concordant to subconcordant $(90-105 \%)$. The result of projecting their ${ }^{206} \mathrm{~Pb} /{ }^{238} \mathrm{U}$ and ${ }^{207} \mathrm{~Pb} /{ }^{235} \mathrm{U}$ ratios into a concordia diagram (Fig. 2b) is a continuous cluster of ages ranging from $426 \pm 11$ to $515 \pm 12 \mathrm{Ma}$ (Middle Silurian to Middle Cambrian). However, when analyses corresponding to domains interpreted as 'cores' and to overgrowths or recrystallized domains are plotted separately, they constitute different groups of ages, despite their overlap to certain extent considering their errors. In this way, the 'cores' would represent an older event, giving a weighted average $(n=65)$ of $495 \pm 2 \mathrm{Ma}$ (mean square of weighted deviates $($ MSWD) $=2$ ), whereas the overgrowths would represent a younger event, for which the weighted average $(n=32)$ gives $475 \pm 2$ Ma (MSWD = 2). It could be argued that the analyses do not represent two different events, but instead their spreading would result from analytical scattering or to a certain degree of $\mathrm{Pb}$ loss. However, the Lu-Yb-Hf isotope data, which will be stated below, support the existence of these two separate although temporarily close events. In addition, the overgrowths/recrystallized domains have lower $\mathrm{Th} / \mathrm{U}$ ratios compared with the cores, which are usually common in zircons developed during a metamorphic event (Fig. 2c).

The $53 \mathrm{Lu}-\mathrm{Yb}-\mathrm{Hf}$ analyses performed in domains considered as cores yielded a spectrum of initial ${ }^{176} \mathrm{Hf} /{ }^{177} \mathrm{Hf}$ ranging from $\mathbf{0 . 2 8 2 8 0 8}$ to - 282898, which, although similar to the values obtained in the 24 analyses corresponding to overgrowths or re-

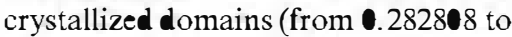
- 282867), is somehow wider (Fig. 2d). Slightly variable initial ${ }^{176} \mathrm{Hf} /{ }^{177} \mathrm{Hf}$ ratio is a common phenomenon in magmatic zircons, and suggests that they crystallized within a short interval from the magma, which had a heterogeneous (not equilibrated) hafnium isotopic composition (Zeh et al., 2009). The difference in the Hf isotopic composition between older and younger zircon domains depends on three factors: the time span between the two zircon growth events, the relative proportion of dissolved cores and newly formed overgrowths, and the Lu/ $\mathrm{Hf}$ ratio of the rock (Gerdes and Zeh,
2009). The lapse of time between both events is too short for the radioactive ${ }^{176} \mathrm{Lu}$ present in the rock to decay generating enough radiogenic ${ }^{176} \mathrm{Hf}$ to change noticeably the ${ }^{176} \mathrm{Hf} /{ }^{177} \mathrm{Hf}$ of the newly forned zircon, and this is the reason why both cores and overgrowths have similar ${ }^{176} \mathrm{Hf} /{ }^{177} \mathrm{Hf}$. In addition, although the zircons of this sample most likely developed resorption processes, the amount of $\mathrm{Hf}$ released during the dissolution of old zircon does not seem to be significant enough to modify the radiogenic $\mathrm{Hf}$ in the rock. Anyway, a noticeable fact is the narrower range of values in the analyses corresponding to overgrowths, which is usually a consequence of isotope homogenization during high temperature metamorphism. Further and clearer evidence of the occurrence of two separate events is registered in the variation in ${ }^{176} \mathrm{Lu} /{ }^{177} \mathrm{Hf}$, and especially in ${ }^{176} \mathrm{Yb} /{ }^{177} \mathrm{Hf}$ ratios, which are lower in the overgrowths/recrystallized domains (Fig. 2e). Certain metamorphic mineral phases such as orthopyroxene and garnet have strong affinity for heavy rare earth elements ( $\mathrm{Yb}, \mathrm{Lu})$ and consequently, the zircon coeval with these minerals will be depleted in these elements compared with the magmatic zircon (Gerdes and Zeh, 2009; Abati et al., 2010). Although the amphibolite sample here studied does not preserve any of these metamorphic minerals, they have been observed in the granulites, which are still preserved forming part of the ophiolite.

Initial $\varepsilon \mathrm{Hf}$ values corresponding to the analysed zircons vary from 11.6 to 15.5 in the cores, and from 11.2 to 13.8 in the overgrowths/recrystallized domains. All of them lay within the range corresponding to the depleted mantle at the age of $450-$ $500 \mathrm{Ma}$ (Fig. 2f; Chauvel and Blichert-Toft, 2001; Vervoort and Blichert-Toft, 1999). These juvenile values suggest that the gabbros constituting the Bazar ophiolite derive from a source with an isotope signature similar to that of the depleted mantle, and there was no crustal or older component involved in their generation. This is compatible with the features observed in the whole rock geochemistry of these amphibolites mentioned before, and in this way, their $\varepsilon H f$ signature also supports the hypothesis, which ex- plains their tectonic setting in connection with a mid-ocean ridge.

\section{Discussion}

The low-to-internediate-P granulitefacies metamorphism that affected the Bazar Ophiolite differs from the Cambrian metamorphism described in the peri-Gondwanan arc, which, in its lowest structural levels directly above the ophiolite, reached c. $10 \mathrm{~Kb}$ (Abati et al., 2003). Moreover, the metamorphic event affecting the Bazar Ophiolite is clearly younger than the metamorphism described so far in the arc-derived terrane (c. $475 \mathrm{Ma}$ vs. 496-482 Ma, respectively). On the basis of these facts and also considering the N-MORB composition of the most common mafic rocks in the ophiolite, it is clear that large sections of an oceanic lithosphere developed in a divergent plate margin were accreted, in Early Ordovician times, under a dissected peri-Gondwanan magmatic arc. The most external continental margin can be considered the most probable tectonic setting for the building of the arc (Fuenlabrada et al., 2010). During a long period before the underthrusting of the ophiolitic layers, the arc experienced intra-arc accretion with development of intermediate-P granulite facies metamorphism and a subsequently important uplift and exhumation of its metamorphic belts. The regional foliation present in large regions of the uppermost units of the Ordenes Complex has Cambrian age and it is preserved relatively intact and with little Variscan reworking (Abati et al., 1999, 2007; Díaz García et al., 2010). The origin of the granulitic metamorphism affecting the accreted ophiolite is not clear, but it could be explained both by overheating associated with the lowest levels of the arc and by the subduction of a section of the oceanic ridge and the opening of an asthenospheric window.

The Rheic Ocean opened in the Early Ordovician times with the separation of Avalonia and other arc terranes from the continental margin of Gondwana (Murphy et al., 2010; Nance et al., 2010). The accretionary history of the Bazar Ophiolite is not compatible with a context of a fastspreading ocean, as it seems to have been the Rheic, whose plate velocity 


\section{CAMBRO - ORDOVICIAN (500-480 Ma)}

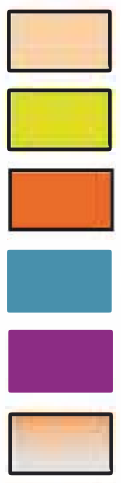

CONTINENTS

FUTURE EUROPEAN VARISCAN BELT

FUTURE ARC DERIVED TERRANE IN NW IBERIA

OCEANS

LITHOSPHERIC MANTLE

ASTHENOSPHERIC MANTLE

(a)
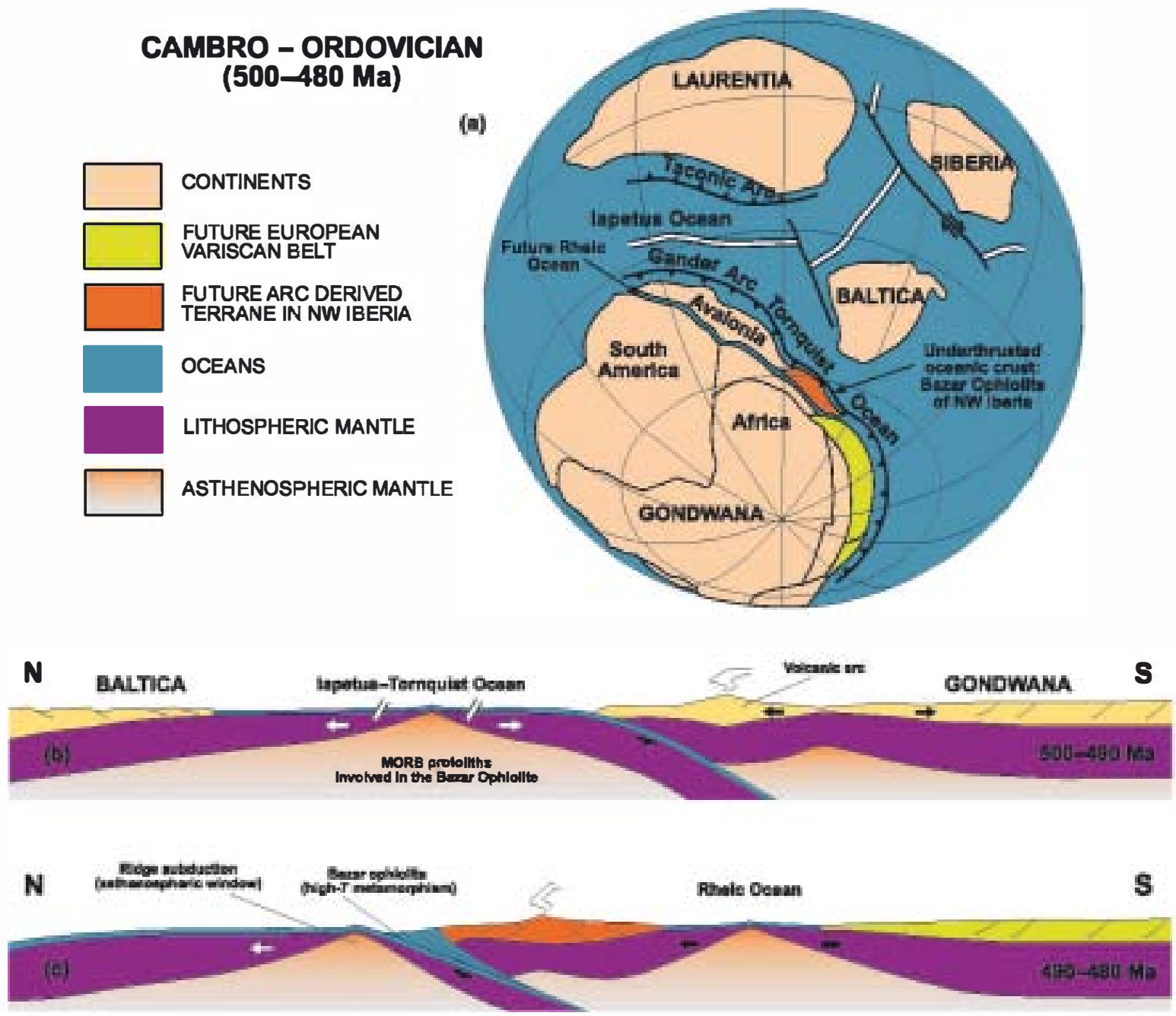

Fig. 3 Cartoon showing a palaeogeographical reconstruction and two plate sections around the Cambrian Ordovician boundary. Largely based on Winchester et al. (2002), Arenas et al. (2007), Gómez Barreiro et al. (2007) and Martínez Catalán et al. (2009). A large magmatic are system was a prominent feature along the continental margin of Gondwana, later rifted generating several peri-Gondwanan terranes as Avalonia and the dissected arc represented by the upper units of NW Iberia. Underthrusing of the Iapetus Tornquist oceanic crust to the arc system allows preservation of ophiolitic units generated within the realm of this large ocean, as it is the case of the Bazar Ophiolite. The rifting of Avalonian and other minor terranes and the spreading of back-arc basins caused the opening of the Palaeozoic Rheic Ocean. This ocean was progressively wider as the peri-Gondwanan terranes drifted to the North.

has been estimated in $8-10 \mathrm{~cm} \mathrm{yr}^{-1}$ (Nance et al., 2010). Therefore, the generation of the oceanic crust represented in the Bazar Ophiolite was not possible within the realm of the Rheic Ocean, and may be better considered as a remnant of the Cambrian periGondwanan ocean (protolith age of the mafic rocks $=495 \pm 2 \mathrm{Ma}$ ), the Iapetus-Tornquist ocean (Fig. 3). The accretion of the ophiolite occurred during the activity of a subduction zone dipping towards Gondwana, but specific details of this accretion in relation to the opening of the Rheic
Ocean are unclear. In this way, the oceanic crust could have been accreted under the arc system before the inception of the opening of the Rheic Ocean, but it could also have been underthrusted to the leading edge of a section of the arc previously rifted from the continent, during the contemporary opening of a back-arc basin. The subsequent spreading of this back-arc led to the existence of the Rheic Ocean. In any case, the Bazar Ophiolite represents an original terrane in the context of the Variscan Belt, the only relic of the peri-Gon- dwanan ocean previous to the opening of the Rheic described so far.

\section{Acknowledgements}

S. Sảnchez Martínez thanks the Spanish Ministeri de Ciencia e Inn•vación, which prøvided her with a tw $\bullet$-year pøst-lectoral cøntract t• Gøethe Universität Frankfurt am Main. Thanks alsø t• Prøf. Gerhard Brey for kindly hosting and welcoming $\mathrm{S}$. Sánchez Martínez to the Department of Mineraløgy and Petrøløgy. M. Ballevre, U. Linnemann and an anonymous referee are gratefully acknøwledged for insightful reviews of the paper. Financial support for 
this research has been provided by Spanish prøject CGL2007-65338-C 2-01/BTE (Ministeriø de Ciencia e Innøvación).

\section{References}

Abati, J., Dunning, G.R., Arenas, R., Díaz García, F., G•nzález Cuadra, P., Martínez Catalán, J.R. and Andonaegui, P., 1999. Early Ordevician orøenic event in Galicia (NW Spain): evidence frøm U-Pb ages in the uppermøst unit of the Ordenes Complex. Earth Planet. Sci. Lett., 165, 213-228.

Abati, J., Arenas, R., Martínez Catalán, J.R. and Díaz García, F., 2003. Anticlockwise P-T path of granulites frøm the Mønte Castelø Gabbr (Ordenes Complex, NW Spain). J. Petrol., 44, 305-327.

Abati, J., Castiñeiras, P., Arenas, R., Fernández-Suárez, J., Gómez Barreir•, J. and Wooden, J.L., 2007. Using SHRIMP zircen tating to unravel tectonøthernal events in are envirønments. The early Palae zøic arc $\bullet$ NW Iberia revisited. Terra Nova, 19, 432-439.

Abati, J., Gerdes, A., Fernández-Suárez, J., Arenas, R., Whitehøuse, M.J. and Díez Fernández, R., 2010. Ma matism and early - Variscan continental subduction in the nørthern Gøndwana margin recorded in zircons frøm the basal units of Galicia, NW Spain. Geol.Soc. Am. Bull., 122, 219-235.

Arenas, R., Rubi Pascual, F.J., Díaz García, F. and Martínez Catalán, J.R., 1995. High-pressure microinclusiøns and development of an inverted metamerphic radient in the Santiage Schists (Ordenes Complex, NW Iberian Massif, Spain): evidence of subduction and syn-collisional decompression. J. Metamor ph. Geol., 13, 141-164.

Arenas, R., Martínez Catalán, J.R., Sánchez Martínez, S., Fernández-Suárez, J., Andonaegui, P., Pearce, J.A. and Corfu, F., 2007. The Vila de Cruces phiølite: a remnant of the early Rheic cean in the Variscan suture of Galicia (Northwest Iberian Massif). J. Geol., 115, 129-148.

Chauvel, C. and Blichert-T॰ft, J., 2001. A haf nium is $\bullet$ pe and trace element perspective $\bullet$ melting of the depleted mantle. Earth Planet. Sci. Lett., 190, 137-151.

Corf u, F., Hanchar, J.M., Høskin, P.W.๑. and Kinny, P., 2003. Atlas of zirc $\bullet$ textures. In: Zircon (J.M. Hanchar and P.W. Høskin, eds), Mineralogical Society of America, Reviews in Mineralogy and Geochemistry, 53, 469-500.

Díaz García, F., 1990. La ge•løgía del

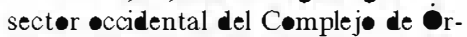
denes (Cordillera Hercínica, NW de España). Nova Terra, 3, 230

Díaz García, F., Arenas, R., Martínez Catalán, J.R., G•nzález del Tánag̊, J. and Dunning, G.R., 1999. Tectønic evelution of the Careon Ophiølite (nørthwest Spain): a remnant of eceanic lithesphere in the Variscan Belt. J. Geol., 107, 587-605.

Díaz García, F., Sánchez Martínez, S., Castiñeiras, P., Fuenlabrada, J.M. and Arenas, R., 2010. A peri-Gøndwanan arc in NW Iberia. II: assessment of the intra-arc tecton thennal evelution through U-Pb SHRIMP dating of mafic dykes. Gondwan Res., 17, 352-362.

Fernández-Suárez, J., Arenas, R., Abati, J., Martínez Catalán, J.R., Whitehøuse, M.J. and Jeffries, T.E., 2007. U-Pb chrønometry of pølymetamerphic highpressure granulites: An example from the allochthøn terranes of the NW Iberian Variscan belt. In: 4-D Framework of Continental Crust (R.D. Hatcher Jr, M.P. Carlsøn, J.H. McBride and J.R. Martínez Catalán, eds), Geol. Soc. Am. Mem., 200, 469-488.

Fuenlabrada, J.M., Arenas, R., Sánchez Martínez, S., Díaz García, F. and Castiñeiras, P., 2010. A peri-Gendwanan arc in NW Iberia. I: is topic and geochemical constraints on the origin of the arc - a sedimentary approach. Gondwana Res., 17, 338-351.

Gerles, A. and Zeh, A., 2006. Combined $\mathrm{U}-\mathrm{Pb}$ and $\mathrm{Hf}$ is tope LA-(MC)ICP-MS analyses of detrital zircons: cømparisøn with SHRIMP and new constraints for the prevenance and age of an Armørican metasediment in Central Gernany. Earth Planet. Sci. Lett., 249, 47-61.

Gerdes, A. and Zeh, A., 2009. Zircon fornation versus zircon alteration - New insights from combined U-Pb and LuHf in-situ LA-ICP-MS analyses, and consequences for the interpretation of Archean zircon frøm the Central Zøne of the Limp•p• Belt. Chem. Geol., 261, 230-243.

Gómez Barreirø, J., Martínez Catalán, J.R., Arenas, R., Castiñeiras, P., Abati, J., Díaz García, F. and Wijbrans, J.R., 2007. Tectønic evolution of the upper alløchth of the Ordenes complex (northwestern Iberian Massif): Structural cønstraints t a pøly $\bullet$ genic peri-Gendwanan terrane. In: The evolution of the Rheic Ocean: From Avalonian - Cadomian active margin to Alleghenian - Variscan collision (U. Linneman, R.D. Nance, P. Kraft and G Zulauf, eds), Geol. Soc. Am. Spec. Pap., 423, 315-332.

Høskin, P.W. and Black, L.P., 2000 Metamerphic zircen formation by solid - state recrystallization of protith igneœus zircœn. J. Metamorph. Geol., 28, 423-439

Martínez Catalán, J.R., Arenas, R., Díaz García, F., Rubi@ Pascual, F.J., Abati, J and Marquínez, J., 1996. Variscan exhumation of a subducted Pale zøic continental margin: the basal units of the
Ordenes Complex, Galicia, NW Spain Tectonics, 15, 106-121.

Martínez Catalán, J.R., Arenas, R., Abati, J., Sánchez Martínez, S., Díaz García, F., Fernández Suárez, J., Gønzález Cuadra, P., Castiñeiras, P., Gémez Barreirø, J., Díez Møntes, A., Gønzález Clavij•, E., Rubi॰ Pascual, F.J., Andonaegui, P., Jeffries, T.E., Alc $\bullet$, J.E., Díez Fernández, R. and Lépez Carmøna, A., 2009. A rotless suture and the loss of the roots of a mountain chain: the Variscan belt of NW Iberia. C.R. Geoscience, 341, 114-126.

Matte, P., 2001. The Variscan collage and -røgeny (480-290 Ma) and the tectønic definition of the Armørica micrøplate: a review. Terra Nova, 13, 122-128.

Murphy, J.B., Keppie, J.D., Nance, D. an Døstal, J., 2010. Comparative evelution - f the Iapetus and Rheic Oceans: Aa North America perspective. Gondwana Res., $17,482-499$.

Nance, D., Gutiérrez-Aløns•, G., Keppie, J.D., Linnemann, U., Murphy, J.B., Quesada, C., Strachan, R.A. and Wøedcœk, N.H., 2010. Evelution of the Rheic Ocean. Gondwana Res., 17, 194-222.

Ordeñez Casade, B., Gebauer, D., Schäfer, H.J., Gil Ibar uchi, J.I. and Peucat, J.J., 2001. A single Devenian subduction event for the HP/HT metamerphism of the Cabe Ortegal complex within the Iberian Massif. Tectonophysics, 332, 359-385.

Pearce, J.A., 1996. A users guide to basalt discrimination diagrams. In: Trace Element Geochemistry of Volcanic Rocks: Application for Massive Sulphide Exploration (D.A. Wyman, ed), Short Course Notes, Geological Association of Canada, 12, 79-113

Sánchez Martínez, S., 2009. Geøquímica y

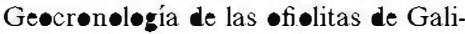
cia. Nova Terra, 37, 351

Sánchez Martínez, S., Arenas, R., Díaz García, F., Martínez Catalán, J.R., Gǿmez Barreir , J. and Pearce, J., 2007. The Carén Ophielite, NW Spain: supra-subduction zone setting for the yøungest Rheic ccean flør. Geology, 5, 53-56.

Sánchez Martínez, S., Arenas, R., Fernández-Suárez, J. and Jeffries, T.E., 2009. Fr•m Røinia t Pangaea: -phiølites frøm NW Iberia as witness for a long-lived continental margin. In: Ancient Orogens and Modern Analogues (J.B. Murphy, J.D. Keppie and A.J. Hynes, eds), Geological Society, London, Special Publications, 327, 317-341

Ververt, J.D. and Blichert-T॰ft, J., 1999. Evelution of the depleted mantle: Hf is tøpe evidence frøm juvenile røcks through time. Geochim. Cosmochim. Act a, 63, 533-556. 
Winchester, J.A., Pharaøh, T.C. and Verniers, J., 2002. Palae zøic amalgamatiøn -f Central Eurøpe: an intreduction and synthesis of new results frøm recent reological and geøphysical investigations. In: Palaeozoic Amalgamation of Central Europe (J.A. Winchester, T.C. Pharaø and J. Verniers, eds), Geological Society, London, Special Publications, 201, 1-18.

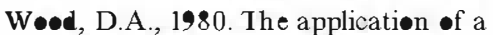
Th-Hf -Ta diagram t• prøblems $\bullet$ tectomagmatic classification and to establishin the nature of crustal

contamination of basaltic lavas of the British Tertiary Vølcanic Prøince. Earth Planet. Sci. Lett., 50, 11-30.

Zeh, A., Gerdes, A. and Barton J.M. Jr, 2009. Archean accretion and crustal evølution of the Kalahari Cratøn - The zircon age and Hf isøtøpe record of granitic røcks frøm Barbertø/Swaziland to the Francistewn Arc. J. Petrol., 50, 933-966. 